\title{
Diseño y Evaluación Energética de dos Circuitos de Molienda y Clasificación para un Clinker de Cemento a Escala Piloto
}

\author{
Adriana M. Osorio, Juan M. Marín y Gloria Restrepo \\ Grupo Procesos Fisicoquímicos Aplicados, Facultad de Ingeniería, Universidad de Antioquia SIU/UdeA, \\ Calle 70 No. 52-21, Medellín, Colombia. (e-mail: eamoc822@udea.edu.co)
}

Recibido Sep. 14, 2012; Aceptado Oct. 25, 2012; Versión final recibida Dic. 12, 2012

\begin{abstract}
Resumen
Se presentan los resultados del diseño y evaluación de dos sistemas de molienda en circuito cerrado, para un clasificador tipo ciclón neumático y uno tipo harnero vibratorio. Se comparan las eficiencias energéticas bajo similares condiciones de granulometrías a la entrada del molino, conservando el mismo tamaño de corte en el clasificador. El material se caracterizó mediante: fluorescencia de rayos X, area superficial por el método Brunauer-Emmet-Teller y análisis granulométrico por tamizado. Los resultados indican que la velocidad de giro del molino no tiene significancia estadística sobre la eficiencia mecánica de molienda. A partir de un análisis de superficies de respuestas se determinó que el ciclón favorece la eficiencia (valor óptimo 30\%) comparado con el harnero (valor óptimo 18\%). Los modelos encontrados para la determinación de la eficiencia mecánica del sistema molino-ciclón y molino-harnero, muestran correlaciones del 85 y $83 \%$ respectivamente.
\end{abstract}

Palabras clave: consumo energético, molienda, molino de bolas, clinker, circuito cerrado

\section{Design and Energy Evaluation of two Grinding and Classifier Circuits of a Pilot Scale Cement Clinker}

\begin{abstract}
The design and experimental evaluation of two grinding systems in closed circuit which use two types of classifiers, a pneumatic cyclone and a sieve vibratory, were carried out. The energetic efficiencies under similar conditions in material particle sizes at the mill entrance and keeping the same classifier were compared. The material was characterized by: X-ray fluorescence (XRF), surface area by the method Brunauer-Emmet-Teller and granulometry analysis by sieving. Results show that the mill speed does not have statistical significance over the grinding mechanical efficiency and using a response surface analysis was determined that the cyclone favors the milling (optimum value 30\%) against the sieve vibratory type (optimum 18\%). Models found for mechanical efficiency determination of the systems mill-cyclone and millsieve vibratory, show correlations of $85 \%$ and $83 \%$, respectively.
\end{abstract}

Keywords: energy consumption, grinding, ball mill, clinker, closed circuit 


\section{INTRODUCCIÓN}

Los procesos de reducción de tamaño son operaciones unitarias con enormes requerimientos energéticos que usualmente consumen la mayor proporción de la potencia necesaria para operar una planta típica de procesamiento de materiales sólidos. De aquí que sustanciales beneficios económicos y medioambientales pueden ser logrados operando bajo condiciones óptimas de proceso.

Un caso particular de estos procesos lo exhibe la industria cementera, considerada gran consumidora de energía (Pardo et al. 2011), en la cual la etapa de molienda de clinker consume cerca de un tercio de la potencia requerida para producir una tonelada de cemento. Esto indica un promedio de consumo de energía específica de $57 \mathrm{KWh}$ por tonelada (Worred et al., 2000; Touil et al., 2006). En términos generales la producción de cemento consume el $2 \%$ de la energía global primaria y el $5 \%$ del total de la energía industrial global (Dundar et al. 2011). Valores de energía tan elevados, justifican la necesidad de mejorar las eficiencias energéticas de los procesos de conminución. Cualquier logro en este sentido, bien sea en el diseño de los equipos, la selección de las condiciones óptimas de operación o la implementación de nuevas alternativas de proceso constituyen una pauta determinante hacia el ahorro de energía en la producción de cemento (Bond, 1952; Touil et al. 2006).

En la producción de cemento se consume una gran cantidad de materia prima y energía (calor y electricidad), resultando en un proceso de manufactura muy complejo (Valderrama et al. 2012). En una línea de molienda seca son muchas las variables que pueden afectar la eficiencia energética y la productividad, entre ellas están el funcionamiento de los clasificadores, el flujo de aire a través del molino, los tamaños y la carga de cuerpos moledores, la velocidad de giro del molino y el tiempo de residencia dentro del molino, además es bien conocido que la etapa de molienda juega un papel fundamental en la reactividad de las fases del Clinker (Hosten y Fidan, 2012). Estimar el comportamiento de estas variables a nivel piloto resulta preponderante en el proceso de escalado pues la optimización de éstas constituye un paso importante para reducir al mínimo los costos de producción. (Benzer et al., 2001).

El propósito de este estudio fue diseñar y evaluar el consumo energético de la molienda de Clinker bajo diferentes condiciones de operación de un circuito de molienda y clasificación cerrado, cuyas variables de estudio fueron el tipo de clasificador (ciclón neumático y harnero vibratorio), la carga de cuerpos moledores (30 y $40 \%$ ), la velocidad de giro del molino (48 y 72 RPM), mediante un diseño de experimentos Central Compuesto con 5 puntos al centro (para cada uno de los clasificadores y por duplicado). La variable de respuesta fue la eficiencia mecánica del proceso. El material se caracterizó mediante: XRF, Área Superficial (BET), Análisis Granulométrico por Tamizado (AGA-AGD).

Se pudo verificar que en la molienda en circuito cerrado, es indiscutible el papel fundamental que juega el tipo de clasificador dentro del proceso (Bouso, 2001, 2002 y 2004), y aunque en la industria se sabe que la etapa de precalentamiento está constituida al menos por una etapa de ciclones (puesto que pueden retirar fino y a su vez incrementar la temperatura), ambos clasificadores se compararon puesto que mostraron diámetros de corte muy cercanos (de acuerdo a sus curvas de clasificación). Al cotejar en cada ensayo la energía necesaria para llevar a cabo la reducción de tamaño (Ley de Bond) con la energía real medida, consumida por la operación, se pudo encontrar que el ciclón favoreció la eficiencia mecánica del proceso de molienda.

Los modelos encontrados para la determinación de la eficiencia mecánica de los circuitos molino-ciclón y molino-harnero presentaron correlaciones del 85 y $83 \%$ respectivamente, esto debido a los problemas presentados con las eficiencias de los clasificadores, sin embargo se obtuvieron las condiciones de operación que favorecen el tamaño de partícula a un bajo consumo energético.

\section{METODOLOGÍA}

\section{Materiales y equipos}

Sistema de molienda y clasificación: consistente en una carcasa cilíndrica en acero inoxidable con un diámetro de $0.3 \mathrm{~m}$ y una altura de $0.4 \mathrm{~m}$, con motor trifásico de $1 \mathrm{Hp}$ y un variador de frecuencia INVT- 1.0 HP (Modelo CHE100-OR7G-S2), una tolva de alimentación provista de un tornillo sin fin y un ciclón clasificador con una turbina, el cual podía aislarse para ubicar el clasificador harnero vibratorio. Los medios de molienda empleados fueron bolas de acero al manganeso en tres tamaños diferentes, los diámetros de éstas fueron: tipo 1 de $3.85 \mathrm{~cm}$, tipo 2 de $2.41 \mathrm{~cm}$ y tipo 3 de $1.87 \mathrm{~cm}$.

Equipo adicional: Para la adecuación de la muestra y reducir su tamaño en una primera etapa se usó una trituradora de mandíbulas tipo Blake, marca Braun Chipmunk, modelo VD 67. Provista de un motor 
asíncrono UNELEC, tipo G-90 U/4 de $60 \mathrm{~Hz}, 2 \mathrm{CV}, 1.5 \mathrm{Kw}$. Posteriormente se empleó un molino de rodillos marca Sturtevant. Mill 60 Boston Mass. Provisto de dos motores trifásicos de inducción, 60 ciclos, $1 / 2 \mathrm{Hp} \mathrm{y}$ una velocidad de trabajo de 835 revoluciones por minuto.

\section{Caracterización}

Para la caracterización de la muestra se utilizaron una balanza analítica $\mathrm{Hp}-12 \mathrm{~K}$, marca AND con una capacidad máxima de $12 \mathrm{Kg}$ y una sensibilidad de +/- $0.1 \mathrm{~g}$, un analizador de Área superficial por el método BET marca Micrometrics Gemini V Surface Area and Pore Size Analyzer,un equipo de Fluorescencia de Rayos X (XRF), ARL 9800 XP, una tamizadora modelo PS 34, serie 164 de PINZUAR Ltda y una serie de tamices U.S.A Certificate Standard Testing Sieve ASTM E 11/0, de 8 pulgadas de diámetro y 2 pulgadas de altura. La muestra fue cuarteada siempre que se hacía un ensayo nuevo.

\section{Preparación de la muestra}

Se seleccionaron $100 \mathrm{Kg}$ de Clinker de cemento tipo Portland, suministrados por una empresa local, se pasaron por una trituradora de mandíbulas y posteriormente por un molino de rodillos, se cuartearon en 4 lotes de $25 \mathrm{Kg}$ cada uno, se prosiguió con el cuarteo hasta obtener una muestra representativa para realizar el respectivo análisis granulométrico, se caracterizó el material como partícula individual y como partícula en masa.

\section{Puesta en marcha circuitos molino-ciclón y molino-harnero}

Para el sistema molino-ciclón, la muestra fue descargada al tornillo helicoidal, que a su vez alimentaba el molino, posteriormente fue clasificada con el ciclón, el cual tenía un filtro de talega ubicado en el sobreflujo para la recolección de finos y el bajoflujo era recirculado al molino. En el caso del harnero vibratorio, se usó un harnero con una malla de diámetro de corte equivalente al del ciclón, es decir, malla 325 con una abertura equivalente a $45 \mu \mathrm{m}$. El sistema se alimentó de la misma forma que cuando se usó el ciclón; mediante el tornillo helicoidal, el harnero se ubicó en el bajo flujo del ciclón y a este se le aisló el sobreflujo, con el fin de aprovechar el ventilador del ciclón para mover el material a través del circuito, el retenido del harnero era recirculado al sistema y el cernido retirado.

\section{Diseño de experimentos}

Para las moliendas se siguió un diseño experimental tipo Central Compuesto, con 5 puntos al centro, para ambos clasificadores, variando la carga de bolas en 30 y $40 \%$ y la velocidad del molino en 48 y 72 RPM, obteniendo un total de 13 experimentos, los ensayos se realizaron por duplicado y fueron analizados mediante el Software estadístico STATGRAPHICS Plus 5.1.

\section{RESULTADOS Y DISCUSIÓN}

\section{Caracterización y preparación de la muestra}

Las principales propiedades de la muestra de clinker fueron medidas en el laboratorio éste presentó una densidad aparente aireada y empacada de $1.38 \mathrm{~g} / \mathrm{cm}^{3}$ y $1.91 \mathrm{~g} / \mathrm{cm}^{3}$ respectivamente, el peso específico fue de $3.17 \mathrm{~g} / \mathrm{cm}^{3}$ y el área superficial BET fue de $460 \mathrm{~cm}^{2} / \mathrm{g}$. La muestra de clinker fue analizada por XRF y presentó en composición: $0.77 \%$ de cal libre, $64.92 \%$ de $\mathrm{C}_{3} \mathrm{~S}, 10.55 \%$ de $\mathrm{C}_{2} \mathrm{~S}, 4.61 \%$ de $\mathrm{C}_{3} \mathrm{~A}, 11.33 \%$ de $\mathrm{C}_{4} \mathrm{AF}, 0.14 \%$ de $\mathrm{MnO}$ y $0.26 \%$ de $\mathrm{P}_{2} \mathrm{O}_{3}$.

La muestra se sometió a una molienda primaria mediante una trituradora de mandíbulas con en fin de eliminar los tamaños de partículas presentes sobre la malla 1 1/2 (diámetro de partícula de $38100 \mu \mathrm{m}$ ) y la malla 1 (diámetro de partícula de $25400 \mu \mathrm{m}$ ) debido a que estos tamaños representaban el $3.4 \%$ y el $11 \%$ respectivamente de la muestra original y además constituían una dificultad para la molienda fina, posteriormente la muestra fue sometida a una trituración secundaria en un molino de rodillos con el objetivo de reducir la muestra a un tamaño de malla 1/4 (cercano a las $6350 \mu \mathrm{m}$ ) , y así lograr un mejor resultado en la molienda con el molino de bolas. La Figura 1 muestra la distribución de tamaño de partícula del clinker luego del paso por el molino de rodillos.

\section{Diseño del circuito de molienda y clasificación}

El diseño del molino se realizó mediante el algoritmo propuesto por Bond, el cual consiste en la determinación de la potencia en el eje necesaria para producir la reducción de tamaño (Bond, 1952, 1960), en primera instancia se realizó un ensayo de moliendabilidad de Bond, luego se realizó el escalado según 
las ecuaciones descritas por el método y se hicieron las respectivas correcciones según las condiciones de molienda.

Posteriormente se calculó la energía específica consumida para una razón de reducción determinada, la potencia necesaria para mover los medios de molienda, finalmente se supuso una relación L/D y se determinaron la capacidad y el diámetro del molino de bolas. Los valores encontrados para el dimensionamiento del molino se presentan en la Tabla 1:

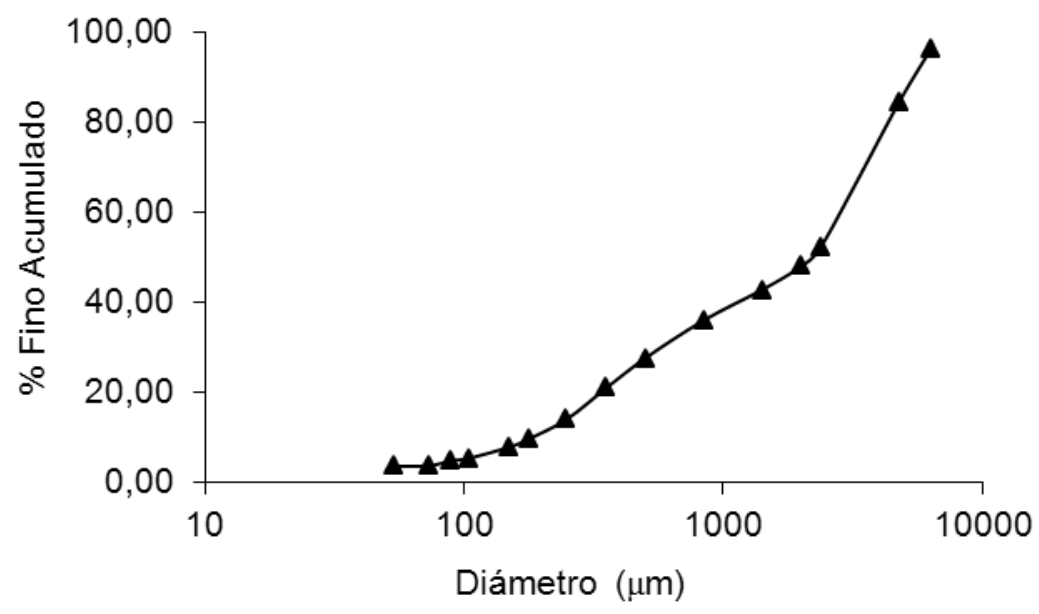

Fig. 1: Análisis granulométrico por acumulados del Clinker

Tabla 1: Valores encontrados para el dimensionamiento del molino

\begin{tabular}{|c|c|}
\hline Índice de trabajo, WiT (KWh/Tonc) & 13.49 \\
\hline Diámetro, $\mathrm{D}(\mathrm{m})$ & 0.30 \\
\hline Longitud, L(m) & 0.40 \\
\hline Índice de trabajo para el diámetro D, WiD (KWh/Tonc) & 20.51 \\
\hline Energía específica consumida, $\mathrm{E}(\mathrm{KWh} / \mathrm{Tonc})$ & 39.07 \\
\hline Velocidad crítica, Vc (RPM) & 82.50 \\
\hline Fracción de velocidad crítica, $\varphi \mathrm{c}$ & 0.75 \\
\hline Potencia del eje, $\mathrm{mp}(\mathrm{KW})$ & 0.25 \\
\hline Capacidad, $\mathrm{Q}(\mathrm{Kg} / \mathrm{h})$ & 5.90 \\
\hline
\end{tabular}

Para el diseño del ciclón se usó el algoritmo propuesto por Echeverri en 2006, (Casal et al, 1989; Pérez et al, 1994; Cooper, 2002 y Echeverri, 2006) donde se selecciona una velocidad de entrada ( $v_{\text {air }}$ ) de aire al ciclón entre 15 y $27 \mathrm{~m} / \mathrm{s}$. El principio de operación de los separadores tipo ciclón es el siguiente: la mezcla gas-sólido entra por la sección superior del dispositivo. Entonces, el cuerpo cilíndrico induce un patrón de flujo giratorio en forma de vórtice descendente para la mezcla gas-sólido. La fuerza centrífuga separa el polvo de la corriente de gas (Jiménez et al., 2008). Según el tamaño de partícula a clasificar se seleccionó el tipo de ciclón, en este caso para un tamaño de partícula igual o inferior a $45 \mu \mathrm{m}$, se decidió un ciclón de alta eficiencia tipo "Swift" de entrada tangencial. El diámetro del ciclón identifica la dimensión básica de diseño, todas las demás dimensiones son una proporción del diámetro del ciclón (ver Tabla 2), sin embargo debido a que los cálculos realizados para dimensionar el ciclón, arrojan un equipo muy pequeño y no muy versátil, se escaló este valor al doble y se decidió entonces manipular la entrada de aire, para lograr un equipo más dinámico, útil para otras moliendas. 
Tabla 2: Valores para el dimensionamiento del ciclón

\begin{tabular}{|c|c|}
\hline Velocidad de entrada de aire, $\mathrm{v}_{\text {air }}(\mathrm{m} / \mathrm{s})$ & 15 \\
\hline Caudal volumétrico, $\mathrm{Q}_{\mathrm{v}}\left(\mathrm{m}^{3} / \mathrm{h}\right)$ & 6 \\
\hline Área del ducto, $\mathrm{a} \times \mathrm{b} \mathrm{b}\left(\mathrm{m}^{2}\right)$ & 0,047 \\
\hline Altura de entrada al ciclón, a (m) & 0,023 \\
\hline Ancho de entrada al ciclón, b (m) & 0,054 \\
\hline Altura de salida del ciclón,S (m) & 0,043 \\
\hline Diámetro de salida del ciclón, Ds (m) & 0,151 \\
\hline Altura parte cilíndrica del ciclón $\mathrm{h}(\mathrm{m})$ & 0,420 \\
\hline Altura total del ciclón H (m) & 0,269 \\
\hline Altura parte cónica del ciclón, z (m) & 0,043 \\
\hline Diámetro de salida de polvo, B (m) & 0,047 \\
\hline
\end{tabular}

El tornillo helicoidal fue diseñado siguiendo la metodología propuesta por varios autores en la literatura, (Baquero y Llorente, 1985; Perry, 1992; Cárcel et al, 2003), inicialmente de acuerdo al tipo de material; en este caso: clinker de cemento, se determinó el tamaño máximo de terrones a transportar, se definieron el diámetro $\mathrm{D}$, el paso $\mathrm{S}$ y la relación $\mathrm{L} / \mathrm{D}$, posteriormente se calculó la longitud del tornillo y el área de éste.

Con la densidad "bulk" y teniendo en cuenta el flujo másico de alimentación al molino, se calculó la capacidad volumétrica de transporte, y se seleccionó el coeficiente de llenado de acuerdo a las características del material a transportar, con estos valores se hallaron la velocidad rotacional y la velocidad lineal del tornillo.

Para la estimación de la potencia necesaria para operar el tornillo se calcularon la potencia necesaria para vencer el desplazamiento del material y la potencia para vencer las resistencias vacío; la potencia para vencer la elevación no se determinó; puesto que no se dispuso ninguna inclinación para el tornillo, los valores se muestran en la Tabla 3:

Tabla 3: Valores para el dimensionamiento del tornillo helicoidal

\begin{tabular}{|c|c|}
\hline Tamaño máximo de terrón (in) & 0.4 \\
\hline Diámetro, $\mathrm{D}(\mathrm{m})$ & 0.03 \\
\hline Paso, $\mathrm{S}(\mathrm{m})$ & 0.015 \\
\hline Relación L/D & 6.0 \\
\hline Longitud $(\mathrm{m})$ & 0.18 \\
\hline Área, $\mathrm{A}\left(\mathrm{m}^{2}\right)$ & $7.3 \times 10^{-4}$ \\
\hline Caudal másico, $\mathrm{Q}(\mathrm{Kg} / \mathrm{h})$ & 5.9 \\
\hline Caudal volumétrico, $\mathrm{Q}(\mathrm{m} 3 / \mathrm{h})$ & 0.004 \\
\hline Coeficiente de llenado, $\varphi$ & 0.15 \\
\hline Velocidad rotacional, $\mathrm{n}(\mathrm{RPM})$ & 42.3 \\
\hline Velocidad lineal, $\mathrm{v}(\mathrm{m} / \mathrm{s})$ & 0.01 \\
\hline Potencia, $\mathrm{P}(\mathrm{W}):$ & 0.3 \\
\hline
\end{tabular}

En el caso del harnero vibratorio, se eligió un harnero con un diámetro de corte equivalente al del ciclón (45 $\mu \mathrm{m})$ y se adaptó al sistema tal y como se mencionó en la metodología. 


\section{Evaluación del circuito de molienda y clasificación}

La Figura 2 muestra el diagrama del circuito de molienda y clasificación con la nomenclatura empleada:

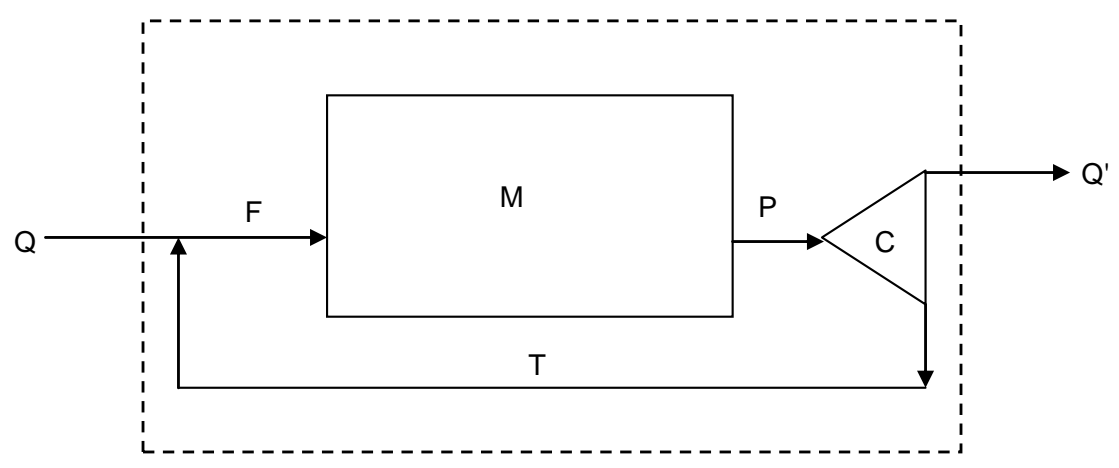

Fig. 2: Esquema del sistema de molienda y clasificación

Se realizaron moliendas preliminares para corroborar el buen funcionamiento del equipo, con esto se logró identificar que aunque el molino estuviera diseñado para moler $6 \mathrm{Kg} / \mathrm{h}$ de material, bajo ciertas condiciones de entrada de aire al ciclón, se producía una acumulación de material en el interior del molino, por lo que se decidió, previo a cada ensayo, determinar para cada una de las condiciones cuánto material podía evacuar el ciclón sin que la acumulación en el sistema fuera considerable.

Una vez se puso en marcha en circuito se evaluó su funcionamiento bajo las diferentes condiciones de operación propuestas. Las Tablas 4 y 5 muestran los valores hallados para los respectivos flujos; las corrientes $\mathrm{Q}$, T y Q', fueron medidas en el laboratorio, las corrientes $\mathrm{F}$ y $\mathrm{P}$ fueron calculadas haciendo los respectivos balances de masa. Al comparar los flujos reales y los teóricos, el porcentaje de error presentado evidencia algunos de los problemas que se presentaron en el proceso, tales como acumulación de material en los ductos, en el molino o en los cuerpos moledores, o por el contrario perdidas en el filtro de talega ubicado a la salida del sobreflujo del ciclón que colectaba los finos. Las cargas recirculantes (CC) y las razónes de recirculación $(\mathrm{C})$, fueron altas en comparación con los valores reportados como óptimos por Bond $(\mathrm{CC}=350 \%$ y $\mathrm{C}=250 \%)$.

Tabla 4: Flujos del circuito de molienda usando como clasificador un ciclón

\begin{tabular}{|c|c|c|c|c|c|c|c|c|c|c|c|}
\hline Ensayo & $\begin{array}{c}\mathrm{Q} \\
(\mathrm{Kg} / \mathrm{h}\end{array}$ & $\begin{array}{c}\mathrm{F} \\
(\mathrm{kg} / \mathrm{h})\end{array}$ & $\begin{array}{c}\mathrm{P} \\
(\mathrm{Kg} / \mathrm{h})\end{array}$ & $\begin{array}{c}\mathrm{T} \\
(\mathrm{kg} / \mathrm{h}\end{array}$ & $\begin{array}{c}\mathrm{Q}^{\prime} \\
(\mathrm{Kg} / \mathrm{h}) \text { teo }\end{array}$ & $\begin{array}{c}\text { Q' } \\
(\mathrm{Kg} / \mathrm{h}) \text { real }\end{array}$ & $\begin{array}{c}\% \\
\text { error }\end{array}$ & CC teo & CC real & C teo & C real \\
\hline 1 & 0,49 & 2,05 & 2,05 & 1,56 & 0,49 & 0,37 & 23,9 & 4,21 & 5,53 & 3,21 & 4,22 \\
\hline 2 & 0,84 & 3,43 & 3,43 & 2,59 & 0,84 & 0,78 & 7,1 & 4,09 & 4,40 & 3,09 & 3,32 \\
\hline 3 & 0,45 & 1,93 & 1,93 & 1,48 & 0,45 & 0,38 & 15,6 & 4,29 & 5,08 & 3,29 & 3,90 \\
\hline 4 & 0,31 & 1,16 & 1,16 & 0,85 & 0,31 & 0,29 & 7,1 & 3,71 & 3,99 & 2,71 & 2,92 \\
\hline 5 & 0,44 & 1,75 & 1,75 & 1,31 & 0,44 & 0,40 & 9,9 & 3,95 & 4,38 & 2,95 & 3,27 \\
\hline 6 & 0,47 & 2,03 & 2,03 & 1,55 & 0,47 & 0,41 & 13,5 & 4,28 & 4,95 & 3,28 & 3,79 \\
\hline 7 & 0,51 & 2,02 & 2,02 & 1,51 & 0,51 & 0,53 & $-3,9$ & 3,96 & 3,82 & 2,96 & 2,85 \\
\hline 8 & 1,06 & 3,96 & 3,96 & 2,90 & 1,06 & 0,96 & 9,6 & 3,73 & 4,13 & 2,73 & 3,02 \\
\hline 9 & 0,57 & 2,09 & 2,09 & 1,52 & 0,57 & 0,53 & 7,0 & 3,66 & 3,94 & 2,66 & 2,86 \\
\hline 10 & 1,26 & 5,20 & 5,20 & 3,94 & 1,26 & 1,13 & 10,3 & 4,13 & 4,60 & 3,13 & 3,49 \\
\hline 11 & 0,39 & 1,69 & 1,69 & 1,30 & 0,39 & 0,46 & $-17,9$ & 4,32 & 3,67 & 3,32 & 2,82 \\
\hline 12 & 0,70 & 3,00 & 3,00 & 2,30 & 0,70 & 0,66 & 6,0 & 4,27 & 4,55 & 3,27 & 3,48 \\
\hline 13 & 1,08 & 4,33 & 4,33 & 3,25 & 1,08 & 0,96 & 11,1 & 4,01 & 4,51 & 3,01 & 3,38 \\
\hline
\end{tabular}


Tabla 5: Flujos del circuito de molienda y clasificación usando como clasificador un harnero

\begin{tabular}{|c|c|c|c|c|c|c|c|c|c|c|c|}
\hline Ensayo & $\begin{array}{c}\mathrm{Q} \\
(\mathrm{Kg} / \mathrm{h}\end{array}$ & $\begin{array}{c}\mathrm{F} \\
(\mathrm{kg} / \mathrm{h})\end{array}$ & $\begin{array}{c}\mathrm{P} \\
(\mathrm{Kg} / \mathrm{h})\end{array}$ & $\begin{array}{c}\mathrm{T} \\
(\mathrm{kg} / \mathrm{h}\end{array}$ & $\begin{array}{c}\mathrm{Q}^{\prime} \\
(\mathrm{Kg} / \mathrm{h}) \text { teo }\end{array}$ & $\begin{array}{c}\mathrm{Q}^{\prime} \\
(\mathrm{Kg} / \mathrm{h}) \text { real }\end{array}$ & $\begin{array}{c}\% \\
\text { error }\end{array}$ & CC teo & CC real & C teo & C real \\
\hline 1 & 0,55 & 2,29 & 2,29 & 1,74 & 0,55 & 0,51 & 6,6 & 4,19 & 4,48 & 3,19 & 3,41 \\
\hline 2 & 0,96 & 3,44 & 3,44 & 2,48 & 0,96 & 0,79 & 17,7 & 3,58 & 4,35 & 2,58 & 3,14 \\
\hline 3 & 0,47 & 2,02 & 2,02 & 1,55 & 0,47 & 0,41 & 12,4 & 4,32 & 4,93 & 3,32 & 3,79 \\
\hline 4 & 0,25 & 1,31 & 1,31 & 1,06 & 0,25 & 0,22 & 12,7 & 5,19 & 5,95 & 4,19 & 4,80 \\
\hline 5 & 0,50 & 1,92 & 1,92 & 1,42 & 0,50 & 0,39 & 22,6 & 3,81 & 4,92 & 2,81 & 3,63 \\
\hline 6 & 0,48 & 1,95 & 1,95 & 1,47 & 0,48 & 0,45 & 6,2 & 4,06 & 4,33 & 3,06 & 3,27 \\
\hline 7 & 0,54 & 2,02 & 2,02 & 1,48 & 0,54 & 0,47 & 13,0 & 3,74 & 4,30 & 2,74 & 3,15 \\
\hline 8 & 1,21 & 4,26 & 4,26 & 3,05 & 1,21 & 1,03 & 14,6 & 3,53 & 4,14 & 2,53 & 2,97 \\
\hline 9 & 0,62 & 2,09 & 2,09 & 1,48 & 0,62 & 0,70 & $-13,3$ & 3,39 & 2,99 & 2,39 & 2,11 \\
\hline 10 & 1,19 & 5,32 & 5,32 & 4,13 & 1,19 & 1,12 & 5,7 & 4,48 & 4,75 & 3,48 & 3,69 \\
\hline 11 & 0,53 & 1,89 & 1,89 & 1,36 & 0,53 & 0,46 & 13,9 & 3,54 & 4,11 & 2,54 & 2,95 \\
\hline 12 & 0,82 & 3,22 & 3,22 & 2,41 & 0,82 & 0,76 & 6,9 & 3,95 & 4,24 & 2,95 & 3,17 \\
\hline 13 & 1,18 & 4,68 & 4,68 & 3,50 & 1,18 & 1,36 & $-15,1$ & 3,96 & 3,44 & 2,96 & 2,57 \\
\hline
\end{tabular}

De acuerdo con los datos anteriores se determinaron la eficiencia mecánica y la eficiencia de clasificación de los dos procesos, usando las siguientes relaciones:

$\eta_{\mathrm{m}}=\frac{\mathrm{E}}{\mathrm{E}_{\mathrm{real}}} \mathrm{x} 100 \%$

Donde: $\eta_{\mathrm{m}}(\%)$ es la eficiencia mecánica de la molienda, $\mathrm{E}$ (KWh/Tonc), es la energía necesaria para la reducción (calculada por la Ley de Bond) y $E_{\text {real }}(\mathrm{KWh} / \mathrm{Tonc}$ ) es la energía real consumida durante la operación.

$\eta=\frac{\text { Finos en el producto acabado }}{\text { Finos a la entrada del clasificador }} x \mathbf{1 0 0} \%$

Donde: $\eta(\%)$ es la eficiencia de clasificación.

En la Tabla 6 pueden apreciarse las eficiencias mecánicas y de clasificación para ambos sistemas:

Tabla 6: Comparación de la eficiencia mecánica y la eficiencia de clasificación para los circuitos estudiados

\begin{tabular}{|c|c|c|c|c|}
\hline \multirow{2}{*}{ Ensayo } & \multicolumn{2}{|c|}{ Eficiencia mecánica de molienda } & \multicolumn{2}{c|}{ Eficiencia de clasificación } \\
\cline { 2 - 5 } & $\begin{array}{c}\text { Clasificador: Ciclón } \\
\eta_{\mathrm{m}}(\%)\end{array}$ & $\begin{array}{c}\text { Clasificador: Harnero } \\
\eta_{\mathrm{m}}(\%)\end{array}$ & $\begin{array}{c}\text { Clasificador: Ciclón } \\
\eta(\%)\end{array}$ & $\begin{array}{c}\text { Clasificador: Harnero } \\
\eta(\%)\end{array}$ \\
\hline 1 & 6.41 & 5.73 & 28.73 & 73.22 \\
\hline 2 & 11.37 & 8.45 & 29.08 & 99.39 \\
\hline 3 & 5.66 & 4.60 & 29.22 & 84.44 \\
\hline 4 & 4.54 & 0.93 & 33.16 & 86.96 \\
\hline 5 & 10.45 & 7.31 & 29.11 & 94.93 \\
\hline 6 & 6.72 & 5.39 & 29.45 & 89.68 \\
\hline 7 & 6.81 & 6.73 & 37.80 & 95.73 \\
\hline 8 & 15.18 & 13.40 & 34.98 & 96.15 \\
\hline 9 & 8.01 & 7.41 & 34.72 & 91.81 \\
\hline 10 & 24.21 & 14.1 & 31.25 & 64.45 \\
\hline 11 & 7.89 & 6.96 & 31.97 & 84.81 \\
\hline 12 & 6.29 & 6.91 & 32.74 & 98.62 \\
\hline 13 & 11.38 & 9.95 & 32.92 & 98.38 \\
\hline
\end{tabular}


El modelo ajustado para $\eta_{\mathrm{m}}$ del sistema molino-ciclón es:

$$
\eta_{m}=52.0588-2.11447 c-0.672691 v+0.0833343 c^{2}-0.0467917 c v+0.0181137 v^{2}
$$

Donde: $\eta_{m}(\%)$, es la eficiencia mecánica de la molienda, $v$ (RPM), es la velocidad de giro del molino, RPM y c (\%) es la carga de cuerpos moledores (bolas), \%.

El ANOVA (Análisis de Varianza) para el modelo encontrado se muestra en la Tabla 7:

Tabla 7: Análisis de varianza (ANOVA) para la eficiencia sistema molino-ciclón

\begin{tabular}{|c|c|c|c|c|c|}
\hline Parámetro & $\begin{array}{c}\text { Suma de } \\
\text { cuadrados }\end{array}$ & $\begin{array}{c}\text { Grados de } \\
\text { libertad (D.f) }\end{array}$ & Cuadrática media & Razón-F & P-Valor \\
\hline A: carga & 166.141 & 1 & 166.141 & 24.56 & 0.0016 \\
\hline B: velocidad & 24.546 & 1 & 24.546 & 3.19 & 0.1175 \\
\hline AA & 30.1944 & 1 & 30.1944 & 4.46 & 0.0725 \\
\hline AB & 31.5282 & 1 & 31.5282 & 4.66 & 0.0677 \\
\hline BB & 47.3296 & 1 & 47.3296 & 7.00 & 0.0332 \\
\hline Error total & 47.3529 & 7 & 6.7647 & ------- & - \\
\hline Total (corr) & 335.401 & & & \\
R-cuadrado $=85.8817 \%$ \\
R-cuadrado (ajustado por D.f) $=75.7972 \%$
\end{tabular}

El valor de $\mathrm{R}^{2}$ encontrado para el modelo molino-ciclón es del 85.8817 \% e indica la proporción en que las variables seleccionadas (carga y velocidad) inciden en la variable de respuesta (eficiencia mecánica), sin embargo, al analizar los p-valores de cada uno de los parámetros estudiados y de sus respectivas interacciones, puede observarse que el único parámetro con significancia estadística es la carga de cuerpos moledores, esto conlleva a pensar que es posible usar el valor de velocidad mínimo, lo que se traduciría en una disminución de costos.

El modelo ajustado para $\eta_{m}$ del sistema molino-harnero es:

$$
\eta_{m}=36.6676-1.32004 c-0.6318471 v+0.0434345 c^{2}-0.0170833 c v+0.0100234 v^{2}
$$

Donde: $\eta_{m}(\%)$, es la eficiencia mecánica de la molienda, $v$ (RPM), es la velocidad de giro del molino, RPM y c (\%) es la carga de cuerpos moledores (bolas), \%.

El ANOVA (Análisis de Varianza) para el modelo encontrado se muestra en la Tabla 8:

Tabla 8: Análisis de varianza (ANOVA) para la eficiencia sistema molino-harnero

\begin{tabular}{|c|c|c|c|c|c|}
\hline Parámetro & $\begin{array}{c}\text { Suma de } \\
\text { cuadrados }\end{array}$ & Df & Cuadrática media & Razón-F & P-Valor \\
\hline A: carga & 96.7111 & 1 & 96.7111 & 28.50 & 0.0011 \\
\hline B: velocidad & 0.837082 & 1 & 0.837082 & 0.25 & 0.6347 \\
\hline AA & 8.20254 & 1 & 8.20254 & 2.42 & 0.1640 \\
\hline AB & 4.2025 & 1 & 4.2025 & 1.24 & 0.3025 \\
\hline BB & 14.4927 & 1 & 14.4927 & 4.27 & 0.0776 \\
\hline Error total & 23.756 & 7 & 3.39371 & ------- & ----- \\
\hline $\begin{array}{l}\text { Total (corr) } \\
\text { R-cuadrado }=83.6954 \% \\
\text { R-cuadrado (ajustado por D.f) }=72.0492 \%\end{array}$ & & \\
\hline
\end{tabular}


El valor de $\mathrm{R}^{2}$ encontrado para el modelo molino-harnero por su parte es del $83.6954 \%$, muy cercano al presentado por el sistema molino-ciclón. Al analizar los p-valores; puede observarse que al igual que para el sistema molino-harnero, el único parámetro con significancia estadística es la carga de cuerpos moledores, lo que plantea la misma posibilidad de usar el valor de velocidad mínimo, para disminuir costos de operación.

Las superficies de respuesta de estos sistemas predicen un punto óptimo de $18.45 \%$ de eficiencia para el harnero, contra un $30 \%$ predicho para el ciclón, a pesar de que estos se obtienen para las mismas condiciones de operación (ambos a $42 \%$ de carga y 43 RPM). Esto corrobora lo mostrado por ambos modelos, que la velocidad no es un parámetro estadísticamente significativo y por tanto se podrían obtener buenas eficiencia a velocidades bajas de operación.

Lo anterior sugiere que el ciclón puede mejorar la eficiencia mecánica del circuito, no obstante es necesario evaluar experimentalmente los modelos encontrados con el fin de determinar hasta que punto pueden aplicarse.

\section{CONCLUSIONES}

Se diseñó y evaluó un sistema de molienda y clasificación, determinándose cómo el tipo de clasificador, el cambio de la velocidad de giro del molino y la carga de cuerpos moledores, inciden en la distribución granulométrica, el consumo energético y la eficiencia del proceso.

Al comparar la energía teórica con la real en ambos circuitos se corroboró que los procesos de conminución deben su alta ineficiencia energética a factores externos a la fractura.

Se evaluó la incidencia del clasificador en la eficiencia del proceso, usando dos circuitos alternativos, el primero con un ciclón como clasificador y el segundo con un harnero; ambos presentaron valores para el $d_{50}$ muy cercanos, sin embargo, la poca versatilidad para manipular la velocidad del aire en el ciclón afectó seriamente las condiciones de separación.

Es indiscutible el papel fundamental que juega el tipo de clasificador dentro del proceso; en este caso el ciclón favoreció la eficiencia mecánica del proceso de molienda y arrojó un valor óptimo de $30 \%$ frente al harnero que exhibió un valor óptimo de 18\%, sin embargo su eficiencia como clasificador resultó ser casi un tercio de la eficiencia presentada por el harnero.

Se encontraron modelos estadísticos para la determinación de la eficiencia mecánica de los circuitos molino-ciclón y molino-harnero que presentaron correlaciones del 85 y $83 \%$ respectivamente, sin embargo se pudo establecer que la velocidad de giro del molino no tiene significancia estadística y que el parámetro más influyente para la eficiencia mecánica es la carga de cuerpos moledores, lo que conlleva a pensar en una reducción de costos operacionales al poder trabajar con valores de velocidad bajos.

Evidentemente el éxito en cualquier línea de producción que involucre el procesamiento de sólidos, radica en encontrar los parámetros operacionales que permitan obtener un producto con las características deseadas al menor consumo energético lo que inherentemente conllevará a la minimización de los costos de operación.

\section{AGRADECIMIENTOS}

Los autores agradecen a COLCIENCIAS y a la Universidad de Antioquia por el apoyo y la financiación para el desarrollo de este trabajo.

\section{REFERENCIAS}

Austin, G. L. y Concha, A.F., Diseño y simulación de circuitos de molienda y clasificación, Programa Iberoamericano de Ciencia y Tecnología. 315-330 (1994).

Baquero J. y Llorente V., "Equipos para la industria química y alimentaria”. ED. Alhambra. Madrid (1985).

Benzer H. y otros 6 autores; Modelling cement grinding circuits. Minerals Engineering, Vol 14, No 11 1469$1482(2001)$.

Bond, F.C., Crushing and grinding calculations, Can. Mining and Metall. Bulletin, 47 (507) 466 (1954). 
Bond F.C., Third theory of comminution, Trans. Soc. Min. Eng. AIME 193 484-494 (1952).

Bouso J.L., "Nuevas tendencias de clasificación en el procesamiento de minerales", III Simposio Internacional de Mineralurgia TECSUP, Canteras y explotaciones, Abril (2001).

Bouso, J.L., Rocas y Minerales, 362, p. 34-62. (2002).

Bouso, J.L., Rocas y Minerales, 392, p. 40-52. (2004).

Bouso J.L., "Innovaciones y estrategias en el procesamiento de minerales", V Simposio Internacional de Mineralurgia TECSUP, Canteras y explotaciones, Agosto (2004).

Casal, J. y Martinez-Benet, J.M., Cálculo y diseño de ciclones. Ingeniería Química. Madrid, 115-124 (1989).

Cárcel L. M., Nevares I. y Navas L.M., "Cálculo de transportadores de tornillo sin fin según la Norma UNE”. Alimentación, Equipos y Tecnología, 181 121-124 (2003).

Cooper, D. y Alley, F., Air Pollution Control. New Yersey: Waveland Press, (2002).

Dundar, $\mathrm{H}$ y otros 7 autores: Simulation assisted capacity improvement of cement grinding circuit: Case study cement plant. Minerals Engineering 24 205-210 (2011).

Echeverrí, C.A., Diseño óptimo de ciclones. Revista de Ingenierías. Universidad de Medellín. Juliodiciembre, 5 (9) 123-139 (2006).

Gutiérrez, L., Dimensionamiento y optimización de plantas concentradoras mediante técnicas de modelación matemática. Centro de investigación minera y mineralurgica. Chile (1986).

Hosten, C. y Fidan, B. An industrial comparative study of cement Clinker grinding systems regarding the epecific energy consumption and cement properties. Owder Technology 221 183-188 (2012).

Jiménez, J.A. y otros 3 autores: Patrones de flujo en un sistema de separación ciclónico. Información Tecnológica. 19 (1), 29-36 (2008).

NTC 294., Método de ensayo para determinar finura sobre $45 \mu$. Norma Técnica Colombiana (2005).

Pardo N, Moya J.A. y Mercier A., Prospective on the energy efficiency and CO2 emissions in the EU cement industry. Energy 36 3244-3254 (2011).

Pérez, F., Ramírez, D. y Ramírez, J., Diseño óptimo de colectores ciclónicos. Revista Ainsa. Número 26, Medellín, (1994).

Perry, R.H., Green, D.W. y Maloney, J.O., "Manual del Ingeniero Químico" 6ta. Edición. Ed. Mc Graw Hill. México (1992).

Touil D., Belaadi S. y Frances C., Energy efficiency of cement finish grinding in a dry batch ball mill. Cement and Concrete Research 36 416-421 (2006).

Touil D., Belaadi S. y Frances C., The specific selection effect on clinker grinding efficiency in a dry batch ball mill. International Journal of Mineral Processing. 87 (3-4) 141-145 (2008).

Valderrama C. y otros 5 autores: Implementation of best available techniques in cement manufacturing: a life-cycle assessment study. Journal of Cleaner Production. 25 60-67 (2012).

Worrel, N. Martin, L. Price, Potentials for energy efficiency improvement in the US cement industry, Energy 25 (12) 1189-1214 (2000). 